\title{
Bupivacaína 0,25\% versus ropivacaína $0,25 \%$ no bloqueio do plexo braquial em cães da raça beagle
}

\section{Bupivacaine 0,25\% versus ropivacaine $0,25 \%$ in brachial plexus block in dogs of beagle breed}

\author{
Thiago Ignácio Wakoff ${ }^{1}$; Rodrigo Mencalha ${ }^{2 *}$; \\ Natália Soares Souza ${ }^{3}$; Carlos Augusto dos Santos Sousa ${ }^{4}$; \\ Mariana do Desterro Inácio e Sousa ${ }^{3}$; Paulo Oldemar Schearer ${ }^{5}$
}

\section{Resumo}

O bloqueio do plexo braquial (BPB) é uma técnica de anestesia regional que possibilita a realização de procedimentos cirúrgicos distais a articulação escapulo-umeral. Este trabalho visou comparar a eficácia da ropivacaína e bupivacaína a $0,25 \%$ sem vasoconstrictor no BPB, guiado por eletroestimulação, em cães. Foram submetidos ao BPB, 13 cães, machos e fêmeas, da raça beagle, utilizando-se bupivacaína ou ropivacaína a $0,25 \%(4 \mathrm{mg} / \mathrm{kg})$, ambos isolados e em períodos distintos. No membro torácico direito foi realizado o bloqueio anestésico e como grupo controle o bloqueio procedeu-se no membro torácico esquerdo com solução de cloreto de sódio $0,9 \%$ no volume correspondente ao do fármaco no membro contralateral. O bloqueio consistiu na localização do nervo radial com o eletroestimulador, onde foi infiltrada a metade do volume anestésico calculado e posteriormente o restante da solução foi administrado sobre o nervo mediano. Foram avaliados os tempos de latência sensitiva e motora e tempos totais de bloqueio motor e sensitivo através da técnica de pinçamentos. No presente estudo, a técnica de eletroestimulação nervosa foi eficaz em 100\% dos animais. A bupivacaína apresentou menor período de latência motora, entretanto, os tempos de latência sensitiva entre os dois fármacos não apresentaram diferenças estatísticas significativas. O tempos de bloqueio com a bupivacaína foi significativamente maior Sinais clínicos característicos de Síndrome de Horner estiveram presentes em 15\% dos animais tratados com bupivacaína. Ademais, dois animais manifestaram sinais de cardiotoxicidade no grupo bupivacaína. O uso da bupivacaína a $0,25 \%$ sem vasoconstrictor na dose de $4 \mathrm{mg} / \mathrm{kg}$ no bloqueio de plexo braquial em cães conferiu maior tempo de analgesia e bloqueio motor, porém, a ropivacaína na mesma dose e concentração mostrou-se livre de efeitos deletérios associados a instabilidade cardiovascular, hemodinâmica e respiratória.

Palavras-chave: Plexo braquial, estimulador, bupivacaína, ropivacaína, cães

\begin{abstract}
The brachial plexus block (BPB) is a regional anesthesia technique which enables the attainment of surgical procedures distal scapulohumeral articulation. This study aimed to compare the efficacy of ropivacaine and bupivacaine $0.25 \%$ without vasoconstrictor in BPB guided by electrical stimulation in dogs. Thirteen male and female beagle dogs underwent a BPB using bupivacaine and ropivacaine $0.25 \%(4 \mathrm{mg} / \mathrm{kg})$, both alone and in different times. The anesthesic block was performed in the right

1 Discente da Universidade Federal Rural do Rio de Janeiro, UFRRJ, Seropédica, RJ. E-mail: thiago_wakoff@hotmail.com

2 Prof. de Anestesiologia, Faculdade de Medicina Veterinária de Valença. E-mail: rmencalha@hotmail.com

3 Discente(s) da UFRRJ, Seropédica, RJ. E-mail: nathalia_scs@hotmail.com; mari_dis@hotmail.com

4 Discente, Faculdade de Medicina Veterinária de Valença. E-mail: augusto.ar@hotmail.com

5 Prof. de Anatomia da UFRRJ, Seropédica, RJ. E-mail: scherer@ufrrj.br

Autor para correspondência
\end{abstract}


forelimb and as control group the block proceeded in the left forelimb using a solution of sodium chloride $0.9 \%$ in volume corresponding to the drug in the contralateral limb. The block was performed after the localization of the radial nerve with the aid of eletrical stimulation, which was infiltrated half the volume of anesthetic calculated and subsequently the remaining solution was administered on the median nerve. We evaluated sensitive and motor latencies and sensitive and motor block total time by clamping technique. In the present study, a technique for electrical stimulation was effective in $100 \%$ of animals. Bupivacaine had lower motor latency period, however, the sensitive latency between the two groups showed no statistically significant differences. In the block total time, bupivacaine obtained time significantly higher. Clinical signs characteristic of Horner's syndrome were present in $15 \%$ of animals treated with bupivacaine. Furthermore, two animals presented signs of cardiotoxicity in bupivacaine group. The use of bupivacaine $(4 \mathrm{mg} / \mathrm{kg}$ ) without vasoconstrictor in dogs brachial plexus block provided longer analgesia and motor blockade, however, ropivacaine at the same dose and concentration was found to be free of deleterious effects associated of cardiovascular instability, hemodynamic and respiratory.

Key words: Brachial plexus, stimulation, bupivacaine, ropivacaine, dogs

\section{Introdução}

Os bloqueios regionais compõem o entendimento da moderna anestesia que tem como objetivo a associação de fármacos e/ou técnicas de modo a explorar efeitos sinérgicos, reduzindo, dessa forma, a quantidade ofertada dos anestésicos gerais sabidamente promotores de efeitos cardiodepressores. A anestesia balanceada, portanto, está cada vez mais presente no cotidiano do anestesiologista propiciando inquestionáveis benefícios como analgesia, relaxamento muscular, hipnose e diminuição do tempo de recuperação anestésico-cirúrgico (ILKIW, 1999; TONNER, 2003; COLUMBANO et al., 2012).

A introdução de fármacos como a ropivacaína na rotina anestésica tem como vantagens um maior tempo de ação, alta potência, baixa capacidade cardiotóxica e baixo potencial arritmogênico em relação a sua antecessora, a bupivacaína (VAINIONPÄÄ et al., 1995; DONY et al., 2000; ZINK; GRAF, 2008; KRUIJT-SPANJER; BAKKER; ABSALOM, 2011). Dessa forma, em associação ao aprimoramento técnico nestes bloqueios, e a utilização do estimulador de nervos periféricos, vêm ocorrendo um aumento no interesse da realização das técnicas de anestesias loco-regionais por médicos veterinários (PASCOE, 1997; FUTEMA et al., 1999; GIULIANO, 2008; VALVERDE, 2008, CASTRO et al., 2009) tendo em vista os sabidos efeitos benéficos de analgesia (LEMKE; DAWSON, 2000; DE MARZO et al., 2012) e supressão do eixo simpato-adrenal (SIBANDA et al., 2006).

A utilização do neuroestimulador vem sendo associada com menor índice de falhas (FANELLI et al., 1999; MAHLER; ADOGWA, 2008), sendo possível identificar precisamente os nervos, podendo estes serem bloqueados individualmente, sem a necessidade da injeção de grandes volumes (RODRÍGUEZ et al., 2004). O bloqueio do plexo braquial é uma técnica de anestesia regional que possibilita a realização de diversos procedimentos cirúrgicos distais a articulação escapulo-umeral (MAHLER; REECE, 2007), e é obtida pela administração perineural do anestésico local neste conjunto de nervos.

O objetivo deste trabalho foi comparar a eficácia da ropivacaína $0,25 \%$ e bupivacaína $0,25 \%$ sem vasoconstrictor no bloqueio do plexo braquial, por via axilar, em cães da raça beagle.

\section{Materiais e Métodos}

Foram utilizados 13 cães (Canis familiaris) da raça beagle, de ambos os sexos, com idade entre um e cinco anos, pesando entre $11 \pm 3 \mathrm{~kg}$, procedentes do Laboratório de Desenvolvimento de Produtos Parasiticidas do Departamento de Parasitologia 
Animal do Instituto de Veterinária da Universidade Federal Rural do Rio de Janeiro.

Para a experimentação, todos os animais após serem submetidos ao jejum prévio de oito horas, e o período de descanso de no mínimo uma semana entre os períodos experimentais, foram submetidos à realização da tricotomia e anti-sepsia dos membros torácicos a serem submetidos ao bloqueio. Procedeu-se a punção da veia cefálica com cateter intravenoso $22 \mathrm{G}^{6}$ e posteriormente a infusão de solução de cloreto de sódio $(\mathrm{NaCl})$ $0,9 \%{ }^{7}$ na velocidade de $10 \mathrm{ml} / \mathrm{kg} / \mathrm{h}$. Na sequencia, procedeu-se a administração de oxigênio por meio de máscara facial por dois minutos, injeção intravenosa de $5 \mathrm{mk} / \mathrm{kg}$ de propofol $^{8}$ e subsequente intubação orotraqueal. Destarte, respeitaram-se os sinais clínicos da indução anestésica e reflexos protetores com intuito de facilitar a realização do bloqueio regional assim como reduzir o estresse do animal durante o procedimento. Nos casos onde os reflexos de proteção demonstraram-se acentuadamente diminuídos antes do término da administração da dose inicial total, a injeção foi interrompida. Entretanto, quando a dose total inicial não foi suficiente ao seu propósito, a administração de mais 2 ou $4 \mathrm{mg} / \mathrm{kg}$ via intravenosa com velocidade de 10 a 20 segundos foi procedida observando a resposta dose-efeito desejada. Logo após a indução, os animais foram submetidos à monitorização multiparamétrica ${ }^{9}$ da pressão arterial não invasiva por método oscilométrico automático com auxílio de manguito na região distal de rádioulna, avaliação eletrocardiográfica, na derivação II do plano frontal, obtido por cabo de cinco vias com garras tipo jacaré e oximetria de pulso por sensor aplicado à língua, mucosa peniana ou vaginal. Após término da instrumentação anestésica, os animais foram submetidos ao bloqueio do plexo braquial

\footnotetext{
${ }^{6}$ Cateter intravenoso 22G - Nipro, Sorocaba, São Paulo, Brasil

7 Cloreto de sódio 0,9\% - Sanobiol, Pouso Alegre, Minas Gerais, Brasil.

8 Diprivan 1\% - AstraZeneca Brasil, Cotia, São Paulo, Brasil.

9 DX 2023 - Dixtal, Santo Amaro, São Paulo, Brasil.
}

com o auxílio do estimulador de nervos periféricos $(\mathrm{ENP})^{10}$. A técnica consistiu na introdução da agulha do ENP"11 $22 \mathrm{G}$ x 2" na região do vazio torácico em direção a articulação costocondral da primeira costela, em busca do nervo radial que responde a sua estimulação com extensão da articulação úmeroradio-ulnar e dos carpos, sendo infiltrada a metade do volume anestésico calculado. Posteriormente foi realizada a busca pelo nervo mediano que responde a sua estimulação com flexão das articulações úmeroradio-ulnar, infiltrando dessa forma o restante do volume anestésico. Iniciou-se a estimulação dos nervos do plexo braquial com uma corrente limiar de $2 \mathrm{~mA}$ e $1 \mathrm{~Hz}$, diminuindo-se gradativamente até obtenção da resposta motora com $0,5 \mathrm{~mA}$. Foram testadas as soluções anestésicas de cloridrato de bupivacaína $^{12}$ e cloridrato de ropivacaína ${ }^{13}$ ambas na concentração de $0,25 \%$ e sem vasoconstrictor, na dose de $4 \mathrm{mg} / \mathrm{kg}$ em dois períodos experimentais distintos: 1) BPB (bloqueio de plexo braquial) c/ ENP (estimulador de nervo periférico) e bupivacaína $0,25 \%$ sem vasoconstrictor na dose de $4 \mathrm{mg} / \mathrm{kg}$ no membro anterior direito e no membro anterior contralateral (grupo controle), BPB c/ ENP e cloreto de sódio $0,9 \%$ no mesmo volume do anestésico utilizado do membro anterior direito. 2) BPB c/ ENP e ropivacaína $0,25 \%$ sem vasoconstrictor na dose de $4 \mathrm{mg} / \mathrm{kg}$ no membro anterior direito e no membro anterior contralateral (grupo controle), BPB c/ ENP e cloreto de sódio 0,9\% no mesmo volume do anestésico do membro anterior direito.

As pressões arteriais sistólica (PAS), diastólica (PAD) e média (PAM) foram mensuradas nos seguintes períodos: imediatamente após indução anestésica (T1); após o término da realização da técnica do bloqueio no membro torácico direito (T2); 15 minutos após o término da realização da técnica do bloqueio no membro torácico direito (T3).

\footnotetext{
${ }^{10}$ Stimuplex - Bbraun, São Gonçalo, Rio de Janeiro, Braisl.

${ }^{11}$ Agulha Stimuplex A50, São Gonçalo, Rio de Janeiro, Braisl.

${ }^{12}$ Neocaína $0,5 \%$ - Cristália, São Paulo, Brasil.

${ }^{13}$ Ropi 1\% - Cristália, São Paulo, Brasil.
} 
Imediatamente após a realização do bloqueio, foi iniciada a avaliação dos seguintes parâmetros em comparação com o membro torácico controle: 1)Tempo de latência sensitiva: desde o término da técnica até a ausência da resposta dolorosa provocada pelo pinçamento da região interdigital e da pele, tanto na porção cranial do rádio quanto da pele na porção cranial e lateral do úmero; 2) Tempo de latência motora: período que compreende desde o término da técnica até a ausência da resposta motora provocada pelo pinçamento da região interdigital e da pele, tanto na porção cranial do rádio quanto da pele na porção cranial e lateral do úmero; 3) Tempo total de bloqueio motor: período que compreende desde o término da latência motora até retorno da ação motora do membro; 4) Tempo total de bloqueio sensitivo: período que compreende desde o término da latência sensitiva até o inicio da resposta dolorosa provocada pelo pinçamento da região interdigital e da pele, tanto na porção cranial do rádio quanto da pele na porção cranial e lateral do úmero.

Todos os pinçamentos propostos anteriormente foram realizados por meio da mesma pinça (hemostática de kocher número quinze), por um período dez segundos cada, com a pressão exercida até alcançar o primeiro dente da cremalheira (ARAÚJO et al., 2001). Para evitar a laceração da pele pela ação dos pinçamentos, um segmento de equipo de fluidoterapia foi cortado e utilizado para recobrir a região denteada da pinça.

A resposta dolorosa aos pinçamentos nos parâmetros tempo de latência sensitiva e tempo total de bloqueio sensitivo foi comprovada pela atitude do animal utilizando-se os seguintes critérios: meneios de cabeça, vocalização, tentativa de mordedura, movimentos de outras partes do corpo e reflexos de defesa como flexão do membro.

Os pinçamentos foram iniciados com intervalo de um minuto nos primeiros 20 minutos e posteriormente respeitaram o intervalo de dois minutos até o tempo de 50 minutos. Após, os pinçamentos respeitaram o intervalo de 10 minutos até o término dos efeitos do bloqueio de plexo braquial.

Os dados referentes ao tempo de latência e tempo total dos bloqueios foram analisados no Software GraphPad Prism 4 utilizando teste T de Student pareado e os dados de pressão arterial sistólica, diastólica e média pelo teste de ANOVA (análise e variância) e teste de médias Tukey com intervalo de confiança de $95 \%$. As diferenças foram consideradas significativas quando $\mathrm{P}<0,05$.

Durante toda fase de experimentação foram respeitados os "Princípios Éticos na Experimentação Animal" postulado pelo Colégio Brasileiro de Experimentação Animal (COBEA), assim como os termos da Lei $n^{\circ} 6.638$, de 08 de maio de 1979 , que estabelece normas para prática científica da vivissecção de animais, e o Decreto ${ }^{\circ} 24.645$ de 10 de julho de 1934 e aprovado pelo conselho de ética da UFRRJ sob processo de número 6577/08.

\section{Resultados}

Os dados obtidos em relação ao tempo de latência sensitiva, latência motora, tempo total de bloqueio sensitivo e tempo total de bloqueio motor de ambos os fármacos estão apresentados nas tabelas 1 e 2 . A bupivacaína apresentou menor período de latência motora $(33,85 \pm 22,27$ versus $53,69 \pm 24,80$ minutos), entretanto, os tempos de latência sensitiva entre os dois fármacos não apresentaram diferenças estatísticas significativas $57,0 \pm 33,25$ minutos (bupivacaína) versus 75,38 $\pm 33,64$ minutos (ropivacaína). Nos tempos totais de bloqueio, a bupivacaína resultou em períodos significativamente mais longos (motor $812,2 \pm 189,7$ versus $346,9 \pm 93,12$ minutos) e (sensitivo $768,5 \pm 184$ versus $296,9 \pm 82,50$ minutos). 
Tabela 1. Valores individuais das latências sensitivas e motoras, tempo total de bloqueio motor e tempo total de bloqueio sensitivo, todos expressos em minutos, com o uso da técnica do bloqueio de plexo braquial com auxílio do estimulador de nervos periféricos obtidos com bupivacaína $0,25 \%$ sem vasoconstrictor em cães da raça beagle.

\begin{tabular}{ccccc}
\hline & $\begin{array}{c}\text { Latência } \\
\text { Sensitiva }\end{array}$ & $\begin{array}{c}\text { Latência } \\
\text { Motora }\end{array}$ & $\begin{array}{c}\text { Tempo Total } \\
\text { Bloqueio Motor }\end{array}$ & $\begin{array}{c}\text { Tempo Total } \\
\text { Bloqueio Sensitivo }\end{array}$ \\
\hline Animal 1 & 70 & 60 & 690 & 650 \\
Animal 2 & 120 & 40 & 670 & 630 \\
Animal 3 & 24 & 20 & 700 & 670 \\
Animal 4 & 22 & 9 & 770 & 680 \\
Animal 5 & 42 & 40 & 740 & 710 \\
Animal 6 & 105 & 85 & 650 & 600 \\
Animal 7 & 70 & 50 & 750 & 710 \\
Animal 8 & 48 & 40 & 820 & 750 \\
Animal 9 & 100 & 15 & 795 & 750 \\
Animal 10 & 44 & 18 & 830 & 720 \\
Animal 11 & 20 & 7 & 703 & 790 \\
Animal 12 & 40 & 34 & 1250 & 1200 \\
Animal 13 & 36 & 22 & 1190 & 1130 \\
\hline Média & 57 & 33,85 & 812,2 & 768,5 \\
\hline Desvio Padrão & 33,35 & 22,27 & 189,7 & 184 \\
\hline
\end{tabular}

Fonte: Elaboração dos autores.

Tabela 2. Valores individuais das latências sensitivas e motoras, tempo total de bloqueio motor e tempo total de bloqueio sensitivo, todos expressos em minutos, com o uso da técnica do bloqueio de plexo braquial com auxílio do estimulador de nervos periféricos obtidos com ropivacaína $0,25 \%$ sem vasoconstrictor em cães da raça beagle.

\begin{tabular}{ccccc}
\hline & $\begin{array}{c}\text { Latência } \\
\text { Sensitiva }\end{array}$ & $\begin{array}{c}\text { Latência } \\
\text { Motora }\end{array}$ & $\begin{array}{c}\text { Tempo Total } \\
\text { Bloqueio Motor }\end{array}$ & $\begin{array}{c}\text { Tempo Total } \\
\text { Bloqueio Sensitivo }\end{array}$ \\
\hline Animal 1 & 50 & 46 & 460 & 410 \\
Animal 2 & 100 & 90 & 250 & 220 \\
Animal 3 & 90 & 60 & 150 & 130 \\
Animal 4 & 36 & 28 & 330 & 290 \\
Animal 5 & 160 & 100 & 250 & 220 \\
Animal 6 & 60 & 40 & 440 & 350 \\
Animal 7 & 38 & 30 & 270 & 230 \\
Animal 8 & 60 & 50 & 450 & 410 \\
Animal 9 & 46 & 28 & 380 & 360 \\
Animal 10 & 90 & 50 & 360 & 340 \\
Animal 11 & 70 & 26 & 370 & 300 \\
Animal 12 & 90 & 70 & 380 & 340 \\
Animal 13 & 90 & 80 & 420 & 260 \\
\hline Média & 75,38 & 53,69 & 346,9 & 296,9 \\
\hline Desvio Padrão & 33,64 & 24,80 & 93,13 & 82,50 \\
\hline
\end{tabular}

Fonte: Elaboração dos autores. 
O tempo de latência motora obtida no grupo não foram observadas diferenças significativas. Os bupivacaína foi significativamente menor $(\mathrm{P}<0,05)$ tempos totais de bloqueio motor e bloqueio sensitivo que o tempo obtido no grupo ropivacaína (figura 1), obtidos foram significativamente superiores entretanto, nos tempos de latência sensitiva (figura 2$) \quad(\mathrm{P}<0,05)$ nos animais do grupo bupivacaína a $0,25 \%$ (figura 3 e 4).

Figura 1. Tempos de latência motora obtidos com o uso da técnica do bloqueio de plexo braquial com auxílio do estimulador de nervos periféricos com a utilização de bupivacaína $0,25 \%$ e ropivacaína 0,25 sem vasoconstrictor com dados representados como média e desvio padrão.

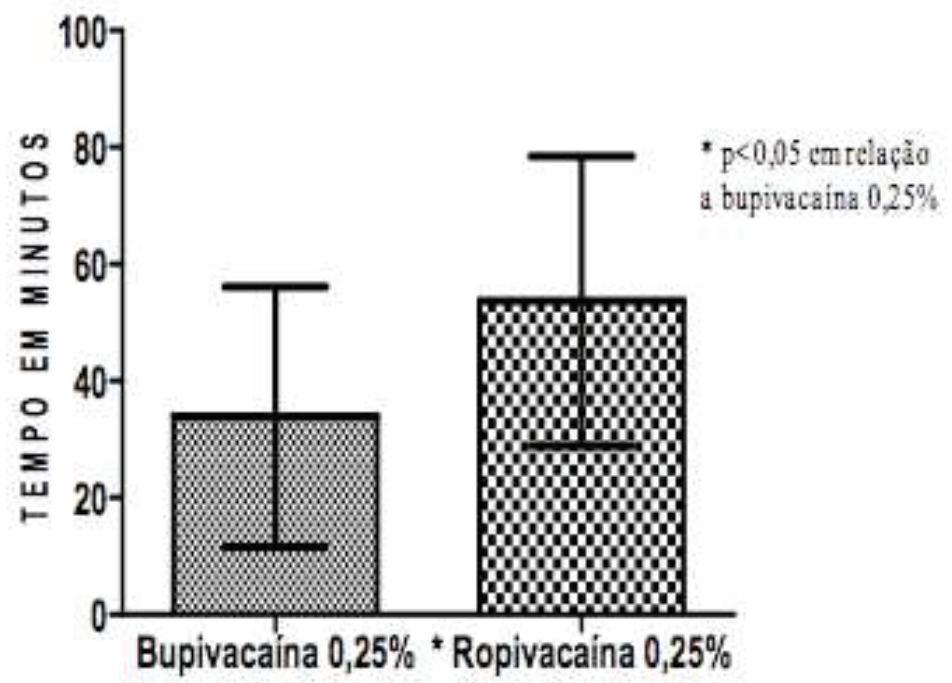

Fonte: Elaboração dos autores.

Figura 2. Tempos de latência sensitiva obtidos com o uso da técnica do bloqueio de plexo braquial com auxílio do estimulador de nervos periféricos com a utilização de bupivacaína $0,25 \%$ e ropivacaína 0,25 sem vasoconstrictor com dados representados como média e desvio padrão.

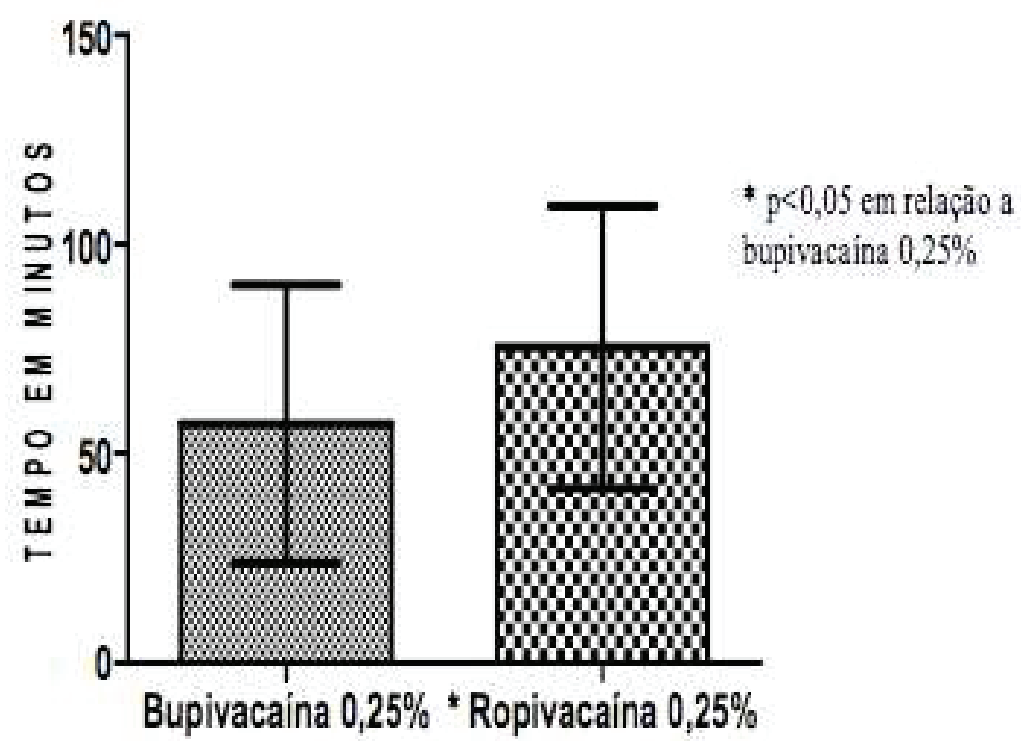

Fonte: Elaboração dos autores. 
Figura 3. Tempos totais de bloqueio motor obtidos com uso da técnica do bloqueio de plexo braquial com auxílio do estimulador de nervos periféricos com a utilização de bupivacaína $0,25 \%$ e ropivacaína $0,25 \%$ sem vasoconstrictor com dados representados como média e desvio padrão.

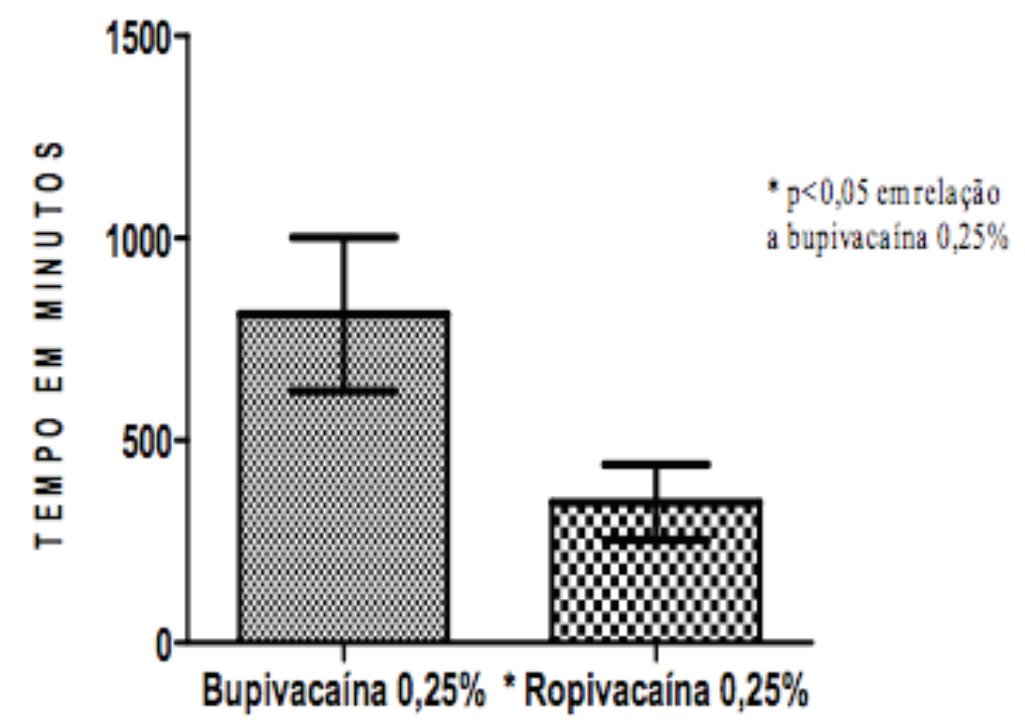

Fonte: Elaboração dos autores.

Figura 4. Tempos totais de bloqueio sensitivo obtidos com uso da técnica do bloqueio de plexo braquial com auxílio do estimulador de nervos periféricos com a utilização de bupivacaína $0,25 \%$ e ropivacaína $0,25 \%$ sem vasoconstrictor com dados representados como média e desvio padrão.

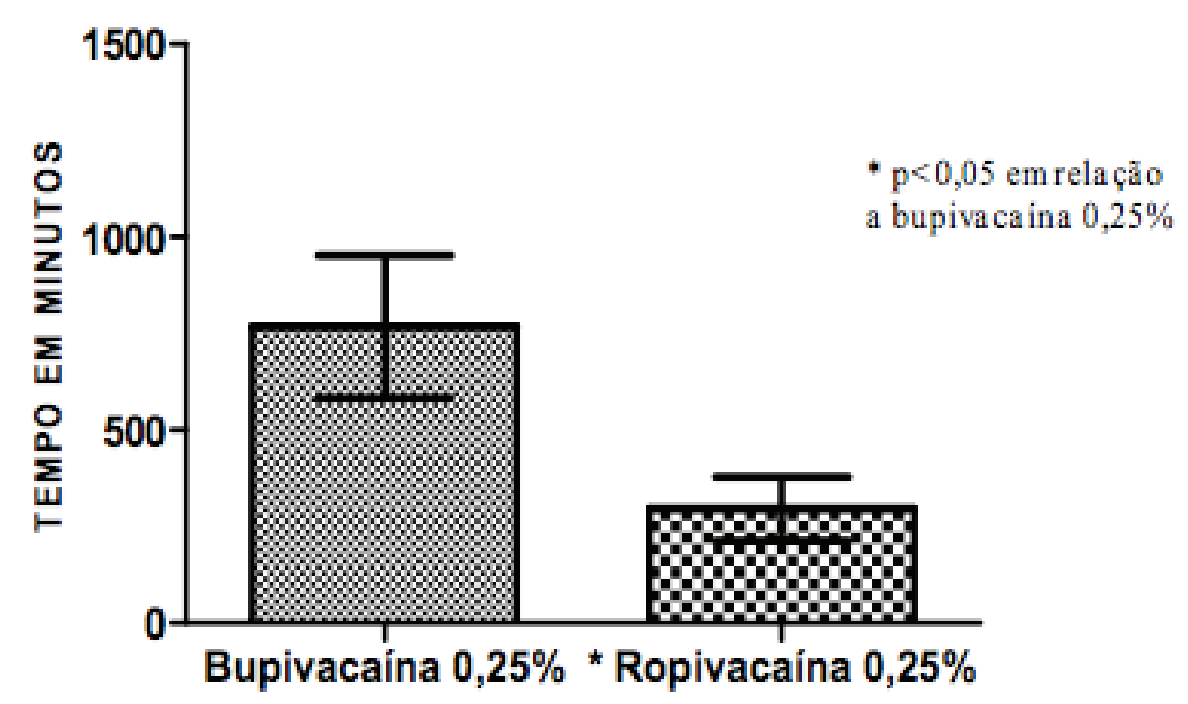

Fonte: Elaboração dos autores.

Os dados obtidos referentes às pressões arteriais sistólica, diastólica e média mensuradas em três tempos distintos (T1, T2 e T3) estão expressos nas tabelas 3 e 4 não havendo diferença significativa entre os grupos. Durante todo o experimento, dos vinte e seis bloqueios realizados no total, cinco animais apresentaram sinais clássicos de síndrome de Horner, sendo quatro desses casos no grupo 
da bupivacaína, ocorrendo resolução espontânea destas complicações após o término do bloqueio. No presente estudo, com ambos os fármacos, todos os bloqueios resultaram em sucesso, ou seja, bloqueio total da capacidade motora e sensitiva na porção distal à articulação úmero-radio-ulnar, portanto houve uma melhora da eficácia da técnica devido à eletroestimulação nervosa sendo eficaz em $100 \%$ dos animais.

Tabela 3. Dados de Pressão Arterial Sistólica (PAS), Pressão Arterial Diastólica (PAD) e Pressão Arterial Média (PAM), em milímetros de mercúrio ( $\mathrm{mmHg}$ ), com utilização de bupivacaína $0,25 \%$ com o uso da técnica de bloqueio do plexo braquial com auxílio do estimulador de nervos periféricos em cães da raça beagle.

\begin{tabular}{|c|c|c|c|c|c|c|c|c|c|}
\hline \multicolumn{10}{|c|}{ BUPIVACAÍNA $0,25 \%$} \\
\hline & \multicolumn{3}{|c|}{ TEMPO I $^{1}$} & \multicolumn{3}{|c|}{ TEMPO II $^{2}$} & \multicolumn{3}{|c|}{ TEMPO III $^{3}$} \\
\hline & PAS & PAD & PAM & PAS & PAD & PAM & PAS & PAD & PAM \\
\hline Animal 01 & 149 & 61 & 86 & 172 & 96 & 110 & 140 & 71 & 103 \\
\hline Animal 02 & 136 & 65 & 90 & 156 & 100 & 120 & 163 & 95 & 116 \\
\hline Animal 03 & 140 & 59 & 86 & 130 & 58 & 80 & 144 & 98 & 116 \\
\hline Animal 04 & 109 & 61 & 81 & 101 & 54 & 74 & 110 & 57 & 77 \\
\hline Animal 05 & 149 & 90 & 110 & 137 & 80 & 105 & 132 & 87 & 99 \\
\hline Animal 06 & 141 & 93 & 111 & 149 & 102 & 120 & 153 & 115 & 125 \\
\hline Animal 07 & 130 & 77 & 99 & 11 & 72 & 89 & 146 & 98 & 114 \\
\hline Animal 08 & 115 & 91 & 99 & 11 & 72 & 89 & 146 & 98 & 114 \\
\hline Animal 09 & 148 & 72 & 108 & 132 & 62 & 99 & 161 & 101 & 123 \\
\hline Animal 10 & 151 & 69 & 102 & 181 & 84 & 115 & 162 & 126 & 130 \\
\hline Animal 11 & 102 & 54 & 79 & 100 & 51 & 77 & 106 & 54 & 73 \\
\hline Animal 12 & 150 & 79 & 105 & 151 & 86 & 106 & 148 & 75 & 97 \\
\hline Animal 13 & 134 & 72 & 98 & 105 & 55 & 76 & 86 & 49 & 52 \\
\hline
\end{tabular}

${ }^{1}$ Imediatamente após indução anestésica e imediata monitoração cardiovascular e respiratória; ${ }^{2}$ Após o término da realização da técnica do bloqueio no membro anterior direito; ${ }^{3} 15$ minutos após o término da realização da técnica no membro anterior direito.

Fonte: Elaboração dos autores. 
Tabela 4. Dados de Pressão Arterial Sistólica (PAS), Pressão Arterial Diastólica (PAD) e Pressão Arterial Média (PAM), em milímetros de mercúrio ( $\mathrm{mmHg}$ ), com utilização de ropivacaína $0,25 \%$ com o uso da técnica de bloqueio do plexo braquial com auxílio do estimulador de nervos periféricos em cães da raça beagle.

\begin{tabular}{|c|c|c|c|c|c|c|c|c|c|}
\hline \multicolumn{10}{|c|}{ ROPIVACAÍNA $0,25 \%$} \\
\hline & \multicolumn{3}{|c|}{ TEMPO I $^{1}$} & \multicolumn{3}{|c|}{ TEMPO II $^{2}$} & \multicolumn{3}{|c|}{ TEMPO III $^{3}$} \\
\hline & PAS & PAD & PAM & PAS & PAD & PAM & PAS & PAD & PAM \\
\hline Animal 01 & 135 & 66 & 86 & 170 & 90 & 106 & 130 & 80 & 100 \\
\hline Animal 02 & 136 & 70 & 82 & 127 & 71 & 96 & 105 & 56 & 74 \\
\hline Animal 03 & 146 & 72 & 97 & 152 & 76 & 106 & 143 & 104 & 113 \\
\hline Animal 04 & 105 & 60 & 82 & 101 & 54 & 74 & 120 & 70 & 100 \\
\hline Animal 05 & 132 & 60 & 86 & 127 & 71 & 96 & 105 & 56 & 74 \\
\hline Animal 06 & 135 & 90 & 100 & 150 & 90 & 110 & 150 & 106 & 112 \\
\hline Animal 07 & 156 & 85 & 109 & 152 & 95 & 119 & 139 & 88 & 110 \\
\hline Animal 08 & 150 & 107 & 123 & 138 & 89 & 107 & 142 & 97 & 110 \\
\hline Animal 09 & 150 & 98 & 119 & 144 & 78 & 103 & 161 & 100 & 124 \\
\hline Animal 10 & 150 & 81 & 97 & 131 & 53 & 92 & 136 & 84 & 101 \\
\hline Animal 11 & 150 & 99 & 116 & 145 & 103 & 116 & 147 & 105 & 115 \\
\hline Animal 12 & 176 & 95 & 129 & 156 & 84 & 112 & 123 & 80 & 92 \\
\hline Animal 13 & 148 & 69 & 91 & 159 & 83 & 106 & 143 & 83 & 103 \\
\hline
\end{tabular}

${ }^{1}$ Imediatamente após indução anestésica e imediata monitoração cardiovascular e respiratória; ${ }^{2}$ Após o término da realização da técnica do bloqueio no membro anterior direito; ${ }^{3} 15$ minutos após o término da realização da técnica no membro anterior direito.

Fonte: Elaboração dos autores.

\section{Discussão}

A técnica utilizada neste estudo foi proposta anteriormente com a finalidade de localizar uma maior quantidade de nervos. Entretanto, neste ensaio experimental limitou-se especificamente aos nervos mediano e radial, com intuito de aprimorar a eficácia, diminuir os erros de técnica e reduzir o tempo de latência (KOSCIELNIAK-NIELSEN; STENS-PEDERSEN; LIPPERT, 1997). Em relação ao risco de neuropraxias causadas pela agulha de punção no bloqueio de plexo braquial, há vantagens na utilização do estimulador de nervos em detrimento da punção “ às cegas ", pois com o auxílio do estimulador é possível localizar o nervo antes que agulha toque no mesmo, o que pode resultar em injúrias no tecido neural (BEATO; CAMOCARDI; IMBELLONI, 2005; IMBELLONI; BEATO; CORDEIRO, 2005). Tais afirmações, corroboram com os presentes resultados onde nenhum animal apresentou lesões ou neuropraxias.
No presente estudo estipulou-se $0,5 \mathrm{~mA}$ como corrente máxima para a administração do anestésico de acordo com estudo realizado outrora em cães por Futema et al. (1999). De acordo com Eifert, Hahnel e Kustermann (1994) a máxima corrente para a injeção do anestésico local deve ser igual ou inferior a $0,5 \mathrm{~mA}$, pois, as falhas do bloqueio ocorridas durante este trabalho foram atribuídas à utilização de $0,6 \mathrm{~mA}$ como referência para a administração do anestésico, enquanto os êxitos foram imputados a utilização de $0,5 \mathrm{~mA}$ como ponto de referência para a administração do anestésico. Sabe-se ainda que quanto menor a miliamperagem, maior a proximidade da agulha ao nervo que se deseja bloquear, sendo a eficiência das técnicas regionais diretamente proporcional à possibilidade de deposição da solução anestésica o mais próximo possível do elemento que se deseja bloquear (RODRÍGUEZ; CARCELLE; ÁLVAREZ, 1996). 
$\mathrm{Na}$ técnica de múltiplas injeções (BARANOWSKI; PITHER, 1990; RODRÍGUEZ; CARCELLE; ÁLVAREZ, 1996), a qual permite que mais de um nervo seja localizado, preconizase que quanto maior a quantidade de nervos localizados durante o bloqueio do plexo braquial mais efetivo será o resultado. No presente estudo, o qual possibilitou a determinação da posição precisa dos nervos radial e mediano por meio de eletroestimulação, houve uma melhora da eficácia devido à posição sintópica destes com os outros nervos que compõem o plexo braquial.

O volume total de anestésico utilizado é fator de grande importância a ser considerado em conjunto com a correta localização e identificação pela resposta motora dos nervos propostos na técnica utilizada (WINNIE; RADONJIC; AKKINENI, 1979). Resultados obtidos em humanos adultos demonstraram que a utilização de bupivacaína $0,25 \%$ ou de ropivacaína $0,25 \%$ no bloqueio de plexo braquial não é eficiente (HICKEY; HOFFMAN; RAMAMURTHY, 1992), confrontando dados do presente estudo o qual evidencia que ambos os fármacos a $0,25 \%$ são capazes de promover o bloqueio regional proposto com sucesso em cães. Da mesma maneira, a ropivacaína $0,2 \%$ e a bupivacaína $0,25 \%$ foram efetivas neste mesmo bloqueio em crianças submetidas a cirurgias de mãos (THORNTON et al., 2003). Tais dados estão possivelmente relacionados com a diferença no calibre das fibras nervosas que compõem este plexo no homem (BOWEN et al., 2004).

De acordo com Baranowski e Pither (1990) nos bloqueios nervosos periféricos os quais envolvem uma série de nervos como o plexo braquial, o bloqueio motor desenvolve-se previamente ao sensitivo, fato este confirmado também no presente estudo onde em todos os animais testados instalouse inicialmente o bloqueio motor, e posteriormente o bloqueio sensitivo.

O período de latência motora obtida no grupo testado pela bupivacaína foi significativamente mais curto do que o do grupo testado com a ropivacaína. Já os tempos de latência sensitiva não divergiram estatisticamente entre os grupos, apesar de os períodos obtidos pelo grupo bupivacaína serem ligeiramente inferiores. Estes resultados confrontam os obtidos em humanos os quais tantos os períodos de latência sensitiva quanto motora foram significativamente mais curtos com a ropivacaína (BERTINI et al., 1999).

As médias dos períodos de latência motora e sensitiva (tempos estes necessários para instalação completa do bloqueio), obtidas no grupo bupivacaína no presente estudo $(33,85 \pm 22,27$ e 57 $\pm 33,35$ minutos respectivamente) foram superiores às obtidas por Futema at al. (1999) que descreveu uma nova técnica deste bloqueio na mesma espécie, o que talvez tenha ocorrido pelo fato de que apesar do presente estudo também utilizar a bupivacaína na dose de $4 \mathrm{mg} / \mathrm{kg}$, houve diferenças na concentração da solução utilizada $(0,25 \%$ versus $0,375 \%)$.

Os tempos de duração total do bloqueio motor e sensitivo foram estatisticamente superiores no grupo bupivacaína, diferindo de estudos em seres humanos os quais, comparando os efeitos clínicos de bupivacaína versus ropivacaína com bloqueio de plexo braquial realizado com diferentes técnicas e concentrações anestésicas obtiveram apenas os tempos de duração total de bloqueio sensitivo estatisticamente iguais (MCGLADE et al., 1998 BERTINI et al., 1999). Especula-se que tal discrepância possa estar relacionada com a diferença de calibre existente entre as fibras motoras de humanos e cães, entretanto, devido a escassez de dados na literatura que comprovem tal afirmação novos estudos são necessários.

No quesito média do período total de bloqueio motor obtido pelo grupo bupivacaína, $812,12 \pm 189,7$ minutos, houve discordância com os valores obtidos em cães e seres humanos onde ambos obtiveram médias abaixo do obtido no presente estudo, mesmo estes tendo utilizado a bupivacaína em maiores concentrações $(0,375 \%$ e $0,5 \%$, respectivamente $)$ 
(BERTINI et al., 1999; FUTEMA et al., 1999). Tal diferença possivelmente ocorreu em função de um maior volume proporcional de infusão dos fármacos supracitados devido a utilização em doses equivalentes e com menores concentrações, possibilitando dessa forma uma maior dispersão dos anestésicos e subsequente área embebida.

No grupo testado pela ropivacaína, a duração de bloqueio motor $(346,9 \pm 93,13)$ e sensitivo $(296,9 \pm 82,50)$ foi significativamente menor do que o do grupo testado com bupivacaína, o que na concepção dos autores possa ter ocorrido pelo fato da ropivacaína além de ser menos potente que a bupivacaína (SIMPSON et al., 2005), ter sido utilizada em baixa concentração, sendo necessário futuros estudos com este anestésico com concentrações maiores para determinação de possíveis aumentos nos tempos totais de bloqueio.

Neste trabalho, manifestações clínicas compatíveis com Síndrome de Horner aconteceram após o bloqueio de plexo braquial em cinco casos durante o estudo, sendo quatro destes casos no grupo bupivacaína, com evolução rápida, as quais regrediram em algumas horas juntamente ao bloqueio sem que houvesse sequelas ou prejuízos posteriores à saúde do animal. Esses dados corroboram com Chambers (1992) o qual relatou sinais ipsilaterais em pacientes humanos submetidos ao bloqueio de plexo braquial com grandes volumes de solução anestésica.

No presente estudo, no grupo testado pela ropivacaína, não houve casos de intoxicação, alterações cardiocirculatórias e hemodinâmicas. Entretanto, no grupo bupivacaína sinais clássicos de cardiotoxicidade foram observadas. Estudos prévios realizados em ratos e cães relataram alterações hemodinâmicas mais drásticas que as observadas neste estudo, que incluíam alterações em sistema nervoso central, bradicardia, bloqueio atrioventricular de segundo grau e hipotensão arterial (DONY et al., 2000; GROBAN et al., 2001). Desta forma, a bupivacaína pode ser utilizada no bloqueio de plexo braquial em cães com cautela e constante monitorização e observação constante de um profissional treinado para tal finalidade, principalmente em animais com instabilidade cardiovascular prévia.

Como discutido acima, em aproximadamente $15 \%$ dos animais testados com bupivacaína houve sinais de absorção maciça de anestésico local 20 a 30 minutos após a realização do bloqueio, nos quais foram observados sinais clínicos semelhantes em todos os cães, dentre eles, destacam-se: hipotensão arterial com pressão sistólica abaixo de $55 \mathrm{mmHg}$ e pressão arterial média abaixo de $40 \mathrm{mmHg}$, bradicardia, depressão respiratória, cianose, mucosas pálidas, letargia, perda de consciência e diminuição do débito urinário. Os animais foram prontamente tratados com expansão volêmica com solução fisiológica $0,9 \%$ via intravenosa na dose de $90 \mathrm{ml} /$ $\mathrm{kg} / \mathrm{h}$ por aproximadamente uma hora e 30 minutos, efedrina na dose de $0,05 \mathrm{mg} / \mathrm{kg}$ intravenosa a cada cinco a 10 minutos e administração de $\mathrm{O}_{2}$ a 100\% após intubação orotraqueal. Casos de hipotensão semelhantes ocorreram nos estudos realizados em animais, onde em ambos os estudos houve correção com infusão de efedrina (RODRÍGUEZ; CARCELLE; ÁLVAREZ, 1996; FUTEMA et al., 1999). No presente estudo, os animais acometidos por essas intercorrências (12 e 13) obtiveram resolução do quadro em torno de seis horas após a injeção, a qual foi efetiva e com os maiores tempos totais de bloqueio motor e sensitivo em comparação aos outros animais testados do mesmo grupo. Tais resultados talvez possam ter ocorridos pela redução da absorção e excreção dos fármacos utilizados, fato estes compatíveis com a instabilidade hemodinâmica apresentada por ambos.

\section{Conclusão}

Ouso dabupivacaína a $0,25 \%$ sem vasoconstrictor na dose de $4 \mathrm{mg} / \mathrm{kg}$ no bloqueio de plexo braquial em cães conferiu maior tempo de analgesia e bloqueio motor, porém, a ropivacaína na mesma dose e 
concentração mostrou-se livre de efeitos deletérios associados à instabilidade cardíaca, hemodinâmica e respiratória.

\section{Referências}

ARAÚJO, I. C.; PROMPERMAYER, L. G.; ANTUNES, F.; SOUZA, A. P.; LOPES, M. A. F. Efeito analgésico do butorfanol na dor somática em gatos anestesiados com propofol. Ciência. Rural, Santa Maria, v. 31, n. 1, p. 6166, 2001.

BARANOWSKI, A. P.; PITHER, C. E. A comparison of three methods of axilary brachial plexus anaesthesia. Anaesthesia, London, v. 45, n. 5, p. 362-365, 1990.

BEATO, L.; CAMOCARDI, G.; IMBELLONI, L. E. Bloqueio do plexo braquial pela via posterior com uso de neuroestimulador e ropivacaína a 0,5\%. Rev. Bras. Anestesiol, Rio de Janeiro, v. 55, n. 4, p. 421-428, 2005.

BERTINI, L.; TAGARIELLO, V.; MANCINI, S.; CIASCHI, A.; POSTERATO, C. M.; BENEDETTO, P. D.; MARTINI, O. $0,75 \%$ and $0,5 \%$ Ropivacaine for axillary brachial plexus block: a clinical comparison with 0,5\% bupivacaine. Reg. Anesth. Pain. Med., Philadelphia, v. 24 , n. 4 , p. $514-518,1999$.

BOWEN, B. C.; PATTANY, P. M.; SARAF-LAVI, E.; MARAVILLA, K. R. The brachial plexus: normal anatomy, pathology, and MR imaging. Neuroimaging Clin. N. Am., Philadelphia, v. 14, n. 1, p. 59-85, 2004.

CASTRO, D. S.; SILVA, M. F.; SHIH, A. C.; MOTA, P. A.; PIRES, M. V. M.; SCHEARER, P. O. Comparison between the analgesic effects of morphine and tramadol delivered epidurally in cats receiving a standardized noxious stimulation. J. Feline Med. Surg, London, v. 11, n. 12, p. 948-953, 2009.

CHAMBERS, W. A. Peripheral nerve damage and regional anesthesia. Br. J. Anaesth., London, v. 69, n. 5, p. 429-430, 1992.

COLUMBANO, N.; SECCI, F.; CAREDDU, G. M.; SOTGIU, G.; ROSSI, G.; DRIESSEN, B. Effects of lidocaine constant rate infusion on sevoflurane requirement, autonomic responses, and postoperative analgesia in dogs undergoing ovariectomy under-based balanced anesthesia. Vet. J., London, v. 193, n. 2, p. 448455, 2012.

DE MARZO, C.; CROVACE, A.; DE MONTE, V.; GRIMALDI, D.; IARUSSI, F.; STAFFIERI, F. Comparison of intra-operative analgesia provided by intravenous regional anesthesia or brachial plexus block for pancarpal arthrodesis in dogs. Res. Vet. Sci., Oxford, v. 99, n. 3, p. 1493-1497, 2012.

DONY, P.; DEWINDE, V.; VANDERICK, B.; CUIGNET, O.; GAUTIER, P.; LEGRAND, E.; LAVAND'HOMME, P.; KOCK, M. D. The comparative toxicity of ropivacaine and bupivacaine at equipotent doses in rats. Anesth. Analg., Cleveland, v. 91, n. 6, p. 1489-149, 2000.

EIFERT, B.; HAHNEL, J.; KUSTERMANN, J. Axillary blockade of brachial plexus. A prospective study of blockade sucess using eletric nerve stimulation. Anaesthesist., Berlin, v. 43, n. 12, p. 780-785, 1994.

FANELLI, G.; CASATI, A.; GARANCINI, P.; TORRI, G. Nerve stimulator and multiple injection technique for upper and lower limb blockade: failure rate, patient acceptance and neurologic complications. Anesth. Analg., Cleveland, v. 88, n. 4, p. 847-852, 1999.

FUTEMA, F.; FANTONI, T. D.; JÚNIOR, J. O. C. A.; CORTOPASSI, S. R. G.; ACAUI, A.; STOPIGLIA, A. J. Nova técnica de bloqueio do plexo braquial em cães. Ciência Rural, Santa Maria, v. 29, n. 1, p. 63-69, 1999.

GIULIANO, E. A. Regional anesthesia as an adjunct for eyelid surgery in dogs. Top Companion Anim Med., New York, v. 23, n. 1, p. 51-56, 2008.

GROBAN, L.; DEAL, D. D.; VERNON, J. C.; JAMES, R. L.; BUTTERWORTH, J. Cardiac resuscitation after incremental overdosage with lidocaine, bupivacaine, levobupivacaine, and ropivacaine in anesthetized dogs. Anesth. Analg., Cleveland, v. 92, n. 1, p. 37-43, 2001.

HICKEY, R.; HOFFMAN, J.; RAMAMURTHY, S. A Comparasion of ropivacaine $0,5 \%$ and bupivacaine $0,5 \%$ for brachial plexus block. Anesthesiology, Philadelphia, v. 74, n. 4, p. 639-642, 1991.

ILKIW, J. E. Balanced anesthetic techniques in dogs and cats. Clin Tech Small Anim Prac., Philadelphia, v. 14, n. 1, p. 27-37, 1999.

IMBELLONI, L. E.; BEATO, L.; CORDEIRO, J. A. Comparison of transarterial and multiple nerve stimulation techniques for axillary block using lidocaine with epinephrine. Rev. Bras. Anestesiol., Rio de Janeiro, v. 55, n. 1, p. $40-49,2005$.

KOSCIELNIAK-NIELSEN, Z. J.; STENS-PEDERSEN, H. L.; LIPPERT, F. K. Readiness for surgery after axillary block: single or multiple injection techniques. Eur J Anesthesiol, Oxford, v. 14, n. 2, p. 164-171, 1997.

KRUIJT SPANJER, M. R.; BAKKER, N. A.;ABSALOM, A. R. Pharmacology in the elderly and newer anaesthesia drugs. Best Pract Res Clin Anaesthesiol, Amsterdam, v. 25, n. 3, p. 355-365, 2011. 
LEMKE, K. A.; DAWSON, S. D. Local and regional anesthesia. Vet. Clin. North Am Small Anim Pract., Philadelphia, v. 30, n. 4, p. 839-857, 2000.

MAHLER, S. P.; ADOGWA, A. O. Anatomical and experimental studies of brachial plexus, sciatic, and femoral nerve-location using peripheral nerve stimulation in the dog. Vet Anaesth Analg., Oxford, v. 35, n. 1, p. 8089, 2008.

MAHLER, S. P.; REECE, J. L. Electrical nerve stimulation to facilitate placement of an indwelling catheter for repeated brachial plexus block in a traumatized dog. Vet Anaesth Analg., Oxford, v. 34, n. 5, p. 365-370, 2007.

MCGLADE, D. P.; KALPOKAS, M. V.; MOONEY, P. H.; CHAMLEY, D.; MARK, A. H.; TORDA, T. A. A comparasion of $0,5 \%$ ropivacaine and $0,5 \%$ bupivacaine for axillary brachial plexus anaesthesia. Anaesth Intensive Care, Sidney, v. 26, n. 5, p. 515-520, 1998.

PASCOE, P. Local and regional anesthesia and analgesia. Semin. Vet. Med. Surg Small Anim., Orlando, v. 12, n. 2, p. 94-105, 1997.

RODRÍGUEZ, J.; BÁRCENA, M.; MUÑIZ-TABOADA, M.; LAGUNILlA, J.; ÁlVAREZ, J. A comparison of single versus multiple injections on the extent of anesthesia with coracoid infraclavicular brachial plexus block. Anesth. Analg., Cleveland, v. 99, n. 4, p. 12251230, 2004.

RODRÍGUEZ, J.; CARCELlE R, J.; ÁlVAREZ, J. Axillary brachial plexus anesthesia: electrical versus cold saline stimulation. Anesth. Analg., Cleveland, v. 83, n. 4, p. 752-754, 1996.
SIBANDA, S.; HUGHES, J. M.; PAWSON, P. E.; KELLY, G.; BELLENGER, C. R. The effects of preoperative extradural bupivacaine and morphine on the stress response in dog sundergoing femoro-tibial joint surgery. Vet Anaesth Analg., Oxford, v. 33, n. 4, p. 246257, 2006.

SIMPSON, D.; CURRAN, M. P.; OLDFIELD, V.; KEATING, G. M. Ropivacaine: a review of its use in regional anaesthesia and acute pain management. Drugs, New York, v. 65, n. 19, p. 2675-2717, 2005.

THORNTON, K. L.; SACKS, M. D.; HALL, R.; BINGHAM, R. Comparasion of $0,2 \%$ ropivacaine and $0,25 \%$ bupivacaine in axillary plexus blocks in paediatric hand surgery. Paediatr Anaesth., Paris, v. 13, n. 5, p. 409$412,2003$.

TONNER, P. H. Balanced anaesthesia today. Best Pract Res Clin Anaesthesiol., Amsterdam, v. 19, n. 3, p. 475484, 2003.

VAINIONPÄÄ, V. A.; HAAVISTO, E. T.; HUHA, T. M.; KORPI, K. J.; NUUTINEN, L. S.; HOLLMÉN, A. I.; JOZWIAK, H. M.; MAGNUSSON, A. A. A clinical and pharmacokinetic comparison of ropivacaine and bupivacaine in axillary plexus block. Anesth. Analg., Cleveland, v. 81, n. 3, p. 534-538, 1995.

VALVERDE, A. Epidural analgesia and anesthesia in dogs and cats. Vet. Clin. North Am Small Anim Pract., Philadelphia, v. 38, n. 6, p. 1205-1230, 2008.

WINNIE, A. P.; RADONJIC, R.; AKKINENI, S. R. Factors influencing distribution of local anesthetic injected into brachial plexus sheath. Anesth. Analg., Cleveland, v. 58, n. 3, p. 225-234, 1979.

ZINK, W.; GRAF, B. M. The toxicity of local anesthetics: the place of ropivacaine and levobupivacaine. Curr Opin Anesthesiol., Philadelphia, v. 21, n. 5, p. 645-650, 2008. 
